\title{
Generation of narrow-band terahertz radiation via optical rectification of femtosecond pulses in periodically poled lithium niobate
}

\author{
Y.-S. Lee, ${ }^{\text {a) }}$ T. Meade, V. Perlin, H. Winful, and T. B. Norris \\ Center for Ultrafast Optical Science, The University of Michigan, Ann Arbor, Michigan 48109-2099
}

\author{
A. Galvanauskas \\ IMRA America, 1044 Woodridge Avenue, Ann Arbor, Michigan 48105
}

(Received 10 February 2000; accepted for publication 13 March 2000)

\begin{abstract}
We demonstrate a promising technique for generating narrow-band terahertz electromagnetic radiation. Femtosecond optical pulses are propagated through a periodically poled lithium-niobate crystal, where the domain length is matched to the walk-off length between the optical and $\mathrm{THz}$ pulses. The bandwidth of the THz wave forms is 0.11 at $1.7 \mathrm{THz}$. Optical rectification gives rise to a $\mathrm{THz}$ wave form which corresponds to the domain structure of the periodically poled lithium niobate. (C) 2000 American Institute of Physics. [S0003-6951(00)03818-3]
\end{abstract}

When an intense optical pulse is incident on a crystal with a nonzero instantaneous second-order optical nonlinearity $\chi^{(2)}$, a nonlinear polarization $P^{(2)}(t)$ is generated which follows the intensity profile $\left|E_{\text {opt }}(t)\right|^{2}$ of the pulse. This process is known as optical rectification, and is widely used for the generation of single-cycle electromagnetic radiation with terahertz (THz) bandwidth. ${ }^{1}$ Ideally, for single-cycle $\mathrm{THz}$ generation, the group velocity of the optical and $\mathrm{THz}$ pulses should be the same in the crystal. In this case, the THz fields radiated from each element of the crystal (proportional to $\left.\partial^{2} P^{(2)} / \partial t^{2}\right)$ add coherently in the forward direction, resulting in radiation of a strong single-cycle pulse. This is often accomplished with a ZnTe crystal and an optical pulse near $800 \mathrm{~nm}^{2}$ If there is a group-velocity mismatch, however, the optical and $\mathrm{THz}$ pulses walk off each other as they propagate through the crystal. As a result, the only nonzero contribution of $P^{(2)}(t)$ to the far-field radiation occurs at the front and back surfaces of the crystal. ${ }^{1}$ If the optical pulse is tightly focused into the crystal, then a single-cycle $\mathrm{THz}$ pulse is radiated in the form of a Cherenkov cone since the optical pulse travels faster than the $\mathrm{THz}$ in the crystal. ${ }^{3}$

In this letter, we describe a different approach to $\mathrm{THz}$ generation by optical rectification, which allows the generation of narrow-band or complex multicycle $\mathrm{THz}$ wave forms. The fundamental idea is to exploit the velocity mismatch between the optical and $\mathrm{THz}$ pulses in a poled nonlinear crystal; the effect is essentially to generate a $\mathrm{THz}$ wave form which corresponds to the domain structure of the poled nonlinear crystal. Phase-matched THz generation in bulk lithium niobate via parametric oscillation has been demonstrated, ${ }^{4}$ and quasi-phase-matched backward parametric oscillation in periodically poled lithium niobate (PPLN) has been proposed..$^{5}$ The technique discussed here is significantly different in that it utilizes the broad bandwidth of femtosecond optical pulses to satisfy a quasi-phase-matching condition enabled by the periodic structure.

The concept was demonstrated using PPLN, for which we have measured the optical index of refraction to be

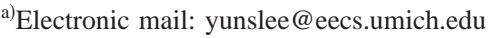

$n_{\text {opt }}=2.3$; the $\mathrm{THz}$ index at 1.7 and $0.67 \mathrm{THz}$ has been reported to be $n_{\mathrm{THz}}=5.2 \pm 0.1$. $^{6}$ It is also confirmed in our $\mathrm{THz}$ wave-form measurements. If the optical pulse duration is $\tau_{p}$, then the optical pulse will lead the THz pulse by $\tau_{p}$ after a walk-off length $l_{w}=c \tau_{p} /\left(n_{\mathrm{THz}}-n_{\text {opt }}\right)$. As illustrated in Fig. 1(a), if the polarization domains of the PPLN reverse sign on a length scale $l_{d} \approx l_{w}$, then the polarization generated in the crystal will radiate a $\mathrm{THz}$ field consisting of $\mathrm{N} / 2$ cycles, where $N$ is the number of domains over the length of the PPLN sample. If the domains are perfectly periodic, narrowband $\mathrm{THz}$ will be generated, with a period $\Delta t_{f}=2 l_{d}\left(n_{\mathrm{THz}}\right.$ $\left.-n_{\text {opt }}\right) / c$. More complex wave forms can be generated with nonperiodically poled structures. ${ }^{7}$ The THz frequency can be varied by changing the domain length $l_{d}$; the optimal conversion of optical to $\mathrm{THz}$ power occurs when the optical pulse width is chosen so $l_{d} \approx l_{w}$.

The experiment was performed using $800 \mathrm{~nm}, 150 \mathrm{fs}$ pulses from a $250 \mathrm{kHz}$ Ti:sapphire regenerative amplifier, ${ }^{8}$ and a $L=1.2 \mathrm{~mm} z$-cut PPLN crystal with domain length $l_{d}$ $=30 \mu \mathrm{m}$. For the reported values of the indices, the calculated $\mathrm{THz}$ frequency should be $1.7 \mathrm{THz}$. The incident optical
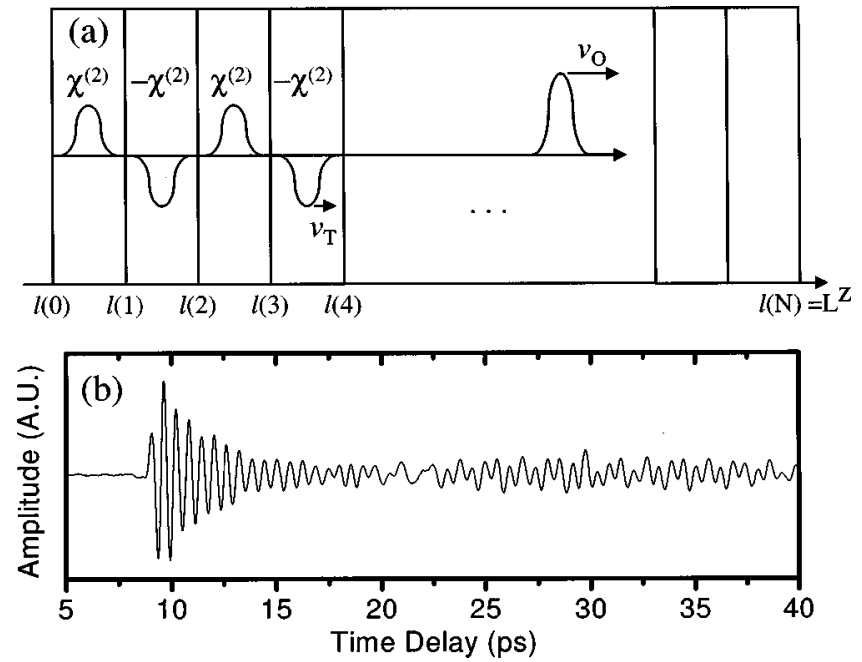

FIG. 1. (a) Schematic diagram of the multiple $\mathrm{THz}$ pulse generation in PPLN. (b) THz wave form via optical rectification in PPLN. 
pulse energy was $40 \mathrm{~nJ}$, focused to a roughly $100 \mu \mathrm{m}$ spot in the crystal. The radiated $\mathrm{THz}$ was collimated with an off-axis parabola and focused into a $1 \mathrm{~mm} \mathrm{ZnTe}$ crystal for electrooptic detection of the $\mathrm{THz}$ field. ${ }^{2}$ The excitation beam was chopped at $50 \mathrm{kHz}$ for lock-in signal detection. An example of the $\mathrm{THz}$ wave form is shown in Fig. 1(b); a damped oscillatory wave form is observed at early times, followed by a more complicated beat pattern at later times.

A simple model may be used to calculate the $\mathrm{THz}$ wave form, assuming plane-wave propagation. The wave equation in the crystal is

$\frac{\partial^{2} E_{\mathrm{THz}}(z, \omega)}{\partial z^{2}}+\epsilon(\omega) \frac{\omega^{2}}{c^{2}} E_{\mathrm{THz}}(z, \omega)=-\frac{4 \pi \omega^{2}}{c^{2}} P^{(2)}(z, \omega)$,

where $\epsilon(\omega)$ is the dielectric function of PPLN. THz loss in the PPLN is modeled through the imaginary part of the refractive index as $\kappa_{T}=g \omega$ ( $g=$ constant). We neglect the dispersion of the optical pulse in the medium. We assume an instantaneous nonlinearity; Fourier transformation of the optical pulse $|E(t)|^{2}$ gives the source term in Eq. (1), which is then integrated to obtain the $\mathrm{THz}$ field $E_{\mathrm{THz}}(z, w)$. For an analytical solution, we may assume a Gaussian input pulse $\left|E_{\text {opt }}(t)\right|^{2}=I_{0} \exp \left(-t^{2} / \tau^{2}\right)$. At the output of the crystal $(z$ $=L$ ), the contribution of the THz field from position $z^{\prime}$ is

$$
\begin{aligned}
E_{\mathrm{THz}}^{\text {local }}\left(z^{\prime}, \omega\right)= & \pm A_{0} \omega^{2} \sqrt{\pi} \tau \exp \left[-\left\{\frac{\tau^{2}}{4}+g / c\left(L-z^{\prime}\right)\right\} \omega^{2}\right. \\
& \left.-i\left(\frac{z^{\prime}}{v_{\mathrm{opt}}}+\frac{L-z^{\prime}}{v_{\mathrm{THz}}}\right) \omega\right]
\end{aligned}
$$

where $v_{\mathrm{opt}}=c / n_{\mathrm{opt}}$ and $v_{\mathrm{THz}}=c / n_{\mathrm{THz}} . A_{0}$ is a constant. The sign of the right-hand side of Eq. (2) is determined by crystal orientation of the domain at $z^{\prime}$. Equation (2) may be Fourier transformed to obtain the time-domain $\mathrm{THz}$ field, and then spatially integrated to give the total output field:

$$
\begin{aligned}
E_{\mathrm{THz}}(z=L, t) & =\frac{1}{L} \int_{0}^{L} E_{\mathrm{THz}}^{\mathrm{local}}\left(z^{\prime}, t\right) d z^{\prime} \\
& =\frac{1}{L} \sum_{i=1}^{N}(-1)^{i-1} \int_{l(i-1)}^{l(i)} E_{\mathrm{THz}}^{\mathrm{local}}\left(z^{\prime}, t\right) d z^{\prime} .
\end{aligned}
$$

Here, the integral has been broken up into a sum of integrals over the $N$ domains in the PPLN, explicitly showing the alternating sign of the polarization in the periodic structure.

A result of the calculation is shown in Fig. 2(a) for 150 fs pulses incident on a PPLN crystal with $L=1.2 \mathrm{~mm}$ and $l_{d}=30 \mu \mathrm{m}$, where loss and domain length fluctuations are neglected. The number of cycles in the THz wave form is $N / 2$. The bandwidth of the spectrum is given essentially by $\delta v / v=2 / N=0.05$. The full width at half maximum of the peak in the power spectrum is $0.077 \mathrm{THz}$ at $1.7 \mathrm{THz}$, which corresponds to $\delta v / v=0.045$. When a phenomenological loss is included $\left(g=7.5 \times 10^{-3} \mathrm{THz}^{-1}\right)$, then the wave form and spectrum are as shown in Figs. 2(b) and 2(e), and the bandwidth is limited by the damping rather than the number of domains. The model accounts quite well for the experimental $\mathrm{THz}$ wave form for early times; the wave form for the initial
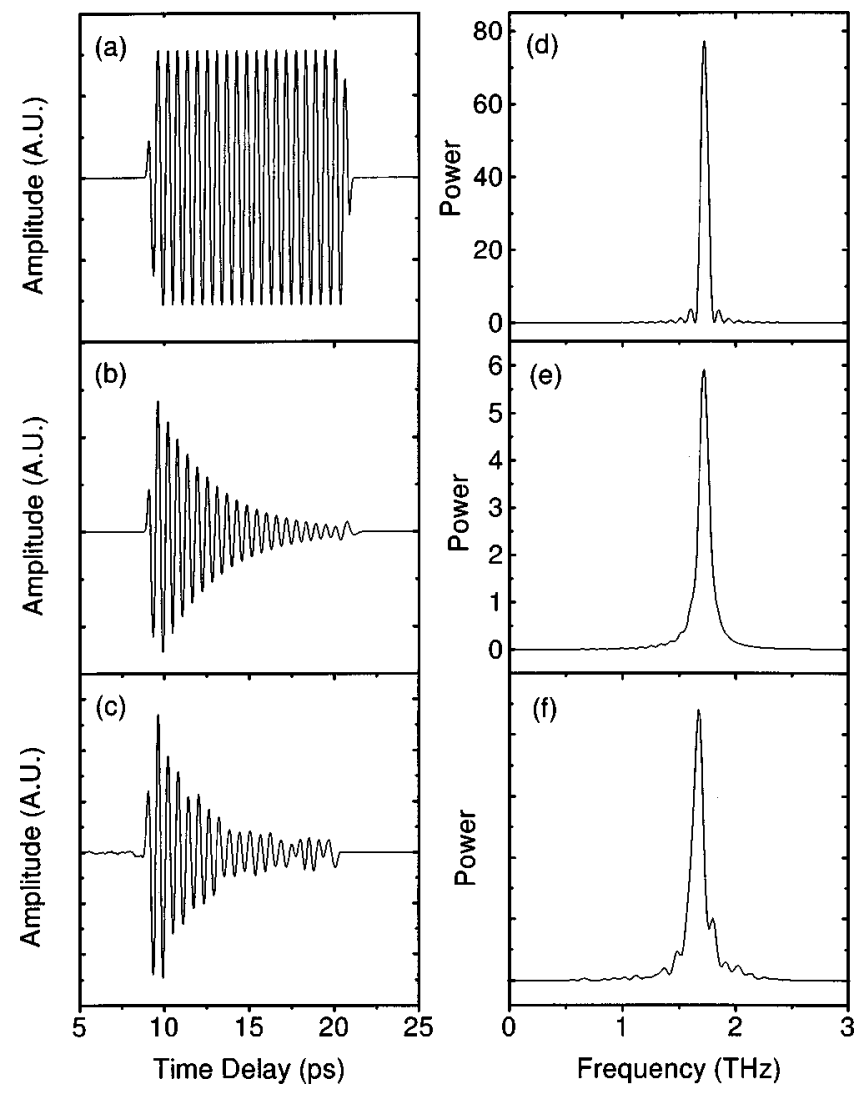

FIG. 2. Simulated and measured $\mathrm{THz}$ wave forms and corresponding power spectra. PPLN domain structure parameters are assumed as $L=1.2 \mathrm{~mm}$ and $l_{d}=30 \mu \mathrm{m}$. (a) and (d): $g=0.0 \mathrm{THz}^{-1}$, (b) and (e): $g=7.5 \times 10^{-3} \mathrm{THz}^{-1}$, and (c) and (f): experimental results.

$12 \mathrm{ps}$ is shown in Fig. 2(c). The spectrum is peaked at 1.7 $\mathrm{THz}$, implying the THz index of refraction to be $n_{\mathrm{THz}}=5.2$. The agreement on bandwidth $(0.11 \mathrm{THz})$ is excellent between simulation and experiment.

The simple model does not, however, account for the wave form for times longer than $12 \mathrm{ps}$. The power spectrum of the full wave form of Fig. 1(b) is shown in Fig. 3(a); a striking feature is the presence of a strong second peak in the spectrum at $0.67 \mathrm{THz}$. This peak may easily be understood to originate from a "backward" wave which propagates in the opposite direction to the optical pulse in the crystal. The optical pulse generates a nonlinear polarization in each domain which can radiate in both the forward and backward direction. Whereas the period of the $\mathrm{THz}$ in the forward direction is given by the difference $\Delta t_{f}=2 l_{d}\left(n_{\mathrm{THz}}-n_{\mathrm{opt}}\right) / c$, it is given in the backward direction by the sum $\Delta t_{b}$ $=2 l_{d}\left(n_{\mathrm{THz}}+n_{\mathrm{opt}}\right) / c$. Inserting the same values for the sample parameters, we find $\left(\Delta t_{b}\right)^{-1}=0.67 \mathrm{THz}$ in good agreement with experiment. We have verified the presence of the backward wave in several samples with different domain lengths $l_{d}$.

Of course, the reason the backward wave is observable in the forward direction is that there is a substantial reflection at the PPLN-air interface. Thus, the backward wave should trail the forward wave in time. This can be seen qualitatively by examination of the wave form in Fig. 1(b). To see this more clearly, however, we need to plot a time-frequency spectrogram to characterize the arrival time of the different frequency components. A convenient way to generate an ap- 

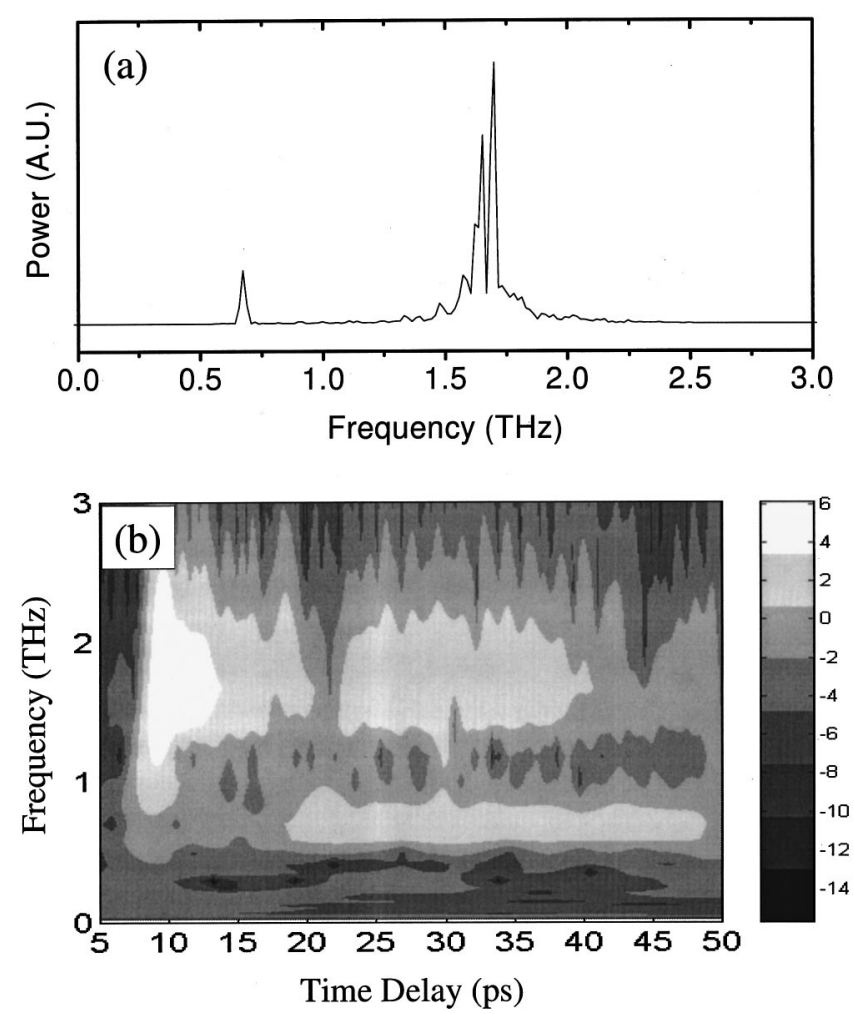

FIG. 3. (a) Power spectrum of THz wave form including backward propagation of $\mathrm{THz}$ waves. (b) Time-frequency spectrogram of the $\mathrm{THz}$ wave form acquired through wavelet decomposition using a Morlet wavelet basis. Brightness indicates the intensity of the frequency component for a given time delay in $\log$ scale.

propriate spectrogram is to perform a wavelet decomposition of the THz wave form. ${ }^{9,10}$ The result (using a Morlet wavelet basis) is shown in Fig. 3(b). The backwards wave clearly arrives at a time corresponding to a wave reflected from the front interface of the PPLN. A component at the forward wave frequency is also observed at a later time; interference between the reflected forward and backward components results in the complicated beating observed in the wave form of Fig. 1(b). The spectrum of the backward wave is narrower ( $\Delta v=0.022 \mathrm{THz}$ ) than that of the forward wave due to the much smaller absorption loss at the lower frequency. The narrow hole in the forward-wave spectrum is likely due to spectral interference between the early and late components around $1.7 \mathrm{THz}$; the detailed origin of this modulation remains the subject of investigation.

What are the prospects for achieving a narrower bandwidth? The absorption loss is expected to be significantly lower if the lithium-niobate crystal is cooled. ${ }^{4}$ This should enable propagation over much longer crystal lengths (perhaps as large as $1 \mathrm{~cm}$ ), thus significantly reducing the bandwidth and increasing the total emitted power proportionately. Poled crystals with lower $\mathrm{THz}$ loss than PPLN may also be developed. For very high-resolution far-infrared spectroscopy, the technique developed by Goyette et al. ${ }^{11}$ may be applied, utilizing very high harmonics of the laser repetition rate, if the $\mathrm{THz}$ can be generated directly with a mode-locked oscillator operating in the $100 \mathrm{MHz}-1 \mathrm{GHz}$ range. The narrow bandwidth of the THz from the PPLN will serve to concentrate the power spectrum around the desired harmonics. Finally, for short-period poled crystals (high $\mathrm{THz}$ frequencies), the bandwidth may be limited by domain length fluctuations. ${ }^{12}$ It is interesting to note that, since the $\mathrm{THz}$ wave form is essentially a direct manifestation of the crystal domain structure, our $\mathrm{THz}$ generation method provides an approach to characterizing the domain structure of poled materials.

This work was supported by ARO under Grant No. DAAH04-96-1-0414 and NSF under Grant No. DMR9729109.

${ }^{1}$ L. Xu, X.-C. Zhang, and D. H. Auston, Appl. Phys. Lett. 61, 1784 (1992).

${ }^{2}$ Q. Wu and X.-C. Zhang, Appl. Phys. Lett. 68, 1604 (1996).

${ }^{3}$ D. H. Auston, K. P. Cheung, J. A. Valdmanis, and D. A. Kleinman, Phys. Rev. Lett. 53, 1555 (1984).

${ }^{4}$ H. Ito, K. Kawase, and J. Shikata, IEICE Trans. Electron Devices E81-C, 264 (1998).

${ }^{5}$ J. Y. Ding and J. B. Khurgin, Opt. Commun. 148, 106 (1998).

${ }^{6}$ H. J. Bakker, S. Hunsche, and H. Kurz, Phys. Rev. B 50, 914 (1994).

${ }^{7}$ K. Fradkin-Kashi and A. Arie, IEEE J. Quantum Electron. 35, 1649 (1999).

${ }^{8}$ T. B. Norris, Opt. Lett. 17, 1009 (1992).

${ }^{9}$ C. Torrence and G. P. Compo, Bull. Am. Meteorol. Soc. 79, 61 (1998).

${ }^{10}$ Wavelet software was provided by C. Torrence and G. P. Compo, and is available at URL: http://paos.colorado.edu/research/wavelets

${ }^{11}$ T. M. Goyette, W. Guo, F. C. De Lucia, J. C. Swartz, H. O. Everitt, B. D. Guenther, and E. R. Brown, Appl. Phys. Lett. 67, 3810 (1995).

${ }^{12}$ G. Rosenman, Kh. Garb, A. Skliar, M. Oron, D. Eger, and M. Katz, Appl. Phys. Lett. 73, 865 (1998). 